\title{
Feasibility of clinicians asking patients about their exposure to occupational hazards: An intervention at five primary care health centres
}

\author{
Rivka Kushner ${ }^{\mathrm{a}}$, Desre M. Kramer ${ }^{\mathrm{a}, *}$ and D. Linn Holness ${ }^{\mathrm{b}, \mathrm{c}}$ \\ ${ }^{a}$ Occupational Cancer Research Centre, Cancer Care Ontario, Toronto, ON, Canada \\ ${ }^{\mathrm{b}}$ Dalla Lana School of Public Health and Department of Medicine, University of Toronto, Toronto, ON, Canada \\ ${ }^{\mathrm{c}}$ Division of Occupational Medicine and Centre for Urban Health Solutions, Li Ka Shing Knowledge Institute, \\ St. Michael's Hospital, Toronto, ON, Canada
}

Received 13 November 2017

Accepted 15 January 2018

\begin{abstract}
. setting. conducted from the feedback. interviews

1. Introduction

Millions of Canadians are potentially exposed to a wide range of known and suspected work-

*Address for correspondence: Desre M. Kramer, Occupational Cancer Research Centre, Cancer Care Ontario, Toronto, ON, Canada. E-mail: desre.kramer2014@gmail.com.
\end{abstract}

BACKGROUND: Ontario's occupational health and safety prevention system has identified a need for the systematic collection of occupational exposure data for ongoing surveillance and targeted prevention initiatives.

OBJECTIVES: To examine the feasibility of collecting occupational exposure information within a primary care clinical

METHODS: Five healthcare centres were recruited. Working patients answered basic occupational exposure questions. Clinicians reviewed the answers with patients. Answers were entered into the patient's electronic medical records (EMR). A knowledge broker supported the health centres throughout the trial with background information and linking to occupational expertise. Interviews with administrators and clinicians examined the usefulness of the survey to primary care, the barriers and facilitators, and sought suggestions for sustaining the practice. A cross-case analysis, framed by a conceptual model, was

RESULTS: Themes highlighted the importance of clinicians and administrator buy-in, the perceived relevance of occupational exposures to primary care clinicians and the patient population, and the need for clinicians to feel confident about the health impact and relevance of occupational exposures to presenting clinical problems.

CONCLUSION: Clinicians ask work exposure-related questions when patients have a health concern that the clinicians suspect may be related to a work exposure. No clear clinical purpose for routinely asking exposure questions emerged.

Keywords: Knowledge transfer implementation, exposure assessment, workplace surveillance, primary healthcare, qualitative

place hazards and risks causing thousands of deaths, disability, and pain. These exposures include chemicals, physical and biological agents, ergonomic and safety hazards, and psychosocial stressors. These exposures, and consequent chronic illnesses, are preventable.

Since 2001 in Canada, there have been more accepted fatality claims related to occupational 
diseases from occupational exposures than related to traumatic injuries and disorders [1]. Occupational disease claims have been steadily increasing over the last dozen years. In 2016, there were 136 occupational disease deaths in Ontario that were compensated, compared to 72 traumatic fatalities [2]. A recent report on the burden of occupational cancer has revealed that workplace exposures to carcinogens such as diesel exhaust, asbestos and silica are causing hundreds of deaths in Ontario each year [3], costing the province millions of dollars in healthcare costs and productivity, and having a negative impact on individuals' quality of life [4].

At the present time in Ontario, there is very limited information being systematically collected on occupational risk factors and exposures in workplaces. There is no central repository of reliable data that can provide us with 'snapshots' of current workplace exposures. There is no early warning system for work-related health conditions, and there are few reliable or accessible sources of information on the location of occupational exposures. The awareness of, and the systematic collection of, surveillance information about these hazards and health effects is needed to accurately assess, reduce, and take prevention measures [5-7].

\subsection{The need to collect occupational exposure data}

At the provincial level, since these exposures are not systematically collected, tracked, measured, or located, researchers cannot establish a basic surveillance system of workplace exposures and risk factors, determine the health impact of present or emerging risks or hazards, or measure the social and personal impact of occupational exposures. Moreover, occupational hazards and risks are not prioritized for prevention measures at the policy or regulation level.

For many years, Ontario's occupational health and safety prevention system has identified that workplace hazard and risk exposure surveillance information needs to be collected. In 2010, the lack of data on occupational exposures and diseases was being highlighted by Ontario's Workplace Safety and Insurance Board (WSIB) as a potential issue. The WSIB adopted an Occupational Disease Response Strategy. The strategy highlighted the need for "appropriate reporting and surveillance mechanisms" [8]. The report emphasized there is presently no effective reporting or surveillance of occupational diseases or exposures and that this is essential for identifying problem areas and monitoring improvements.

In December 2013, the Ontario Ministry of Labour launched its Integrated Health and Safety Strategy to "improve the delivery of workplace health and safety" [9]. In 2017, the Ontario Occupational Disease Action Plan (ODAP) and an Occupational Disease Framework were announced [10]. Building on the past, one of the initiatives listed under the ODAP is to systematically collect workplace exposure data, and create a strategy to embed 'Occupation' into patients' electronic medical records (EMRs) [10].

\subsection{Targeting primary care as the collectors of occupational data}

Targeting primary care clinicians to collect surveillance data on workplace exposures in their patients' EMRs is a logical proposal. Primary care providers see patients with work-related problems [11]. They are important knowledge brokers for workers at risk. They play a central role in the workers' compensation process - recognizing work-related injuries and illnesses, providing information to workers' compensation boards about the nature of injuries or illnesses, and providing treatment for sick and injured workers [11].

Clinicians could be collecting this information as part of the information they collect on patients' health history. Work and working conditions are major social determinants of health. Clinicians often ask about family, family history, lifestyle, and income or job. If questions about occupational exposures were asked in a systematic way, then the information could easily be entered in the EMR and could be referenced in future health appointments. Furthermore, if the electronic medical records across primary care were linked (there are 17 different certified EMR platforms presently), then a system could be established to collect and analyze this data for surveillance, leading to evidence-based prevention measures at the provincial level.

However, despite these advantages, previous research has suggested that although primary care clinicians think it is important to record patients' occupations, they infrequently and inconsistently collect occupational information from their patients $[12,13]$. Barriers to collecting this information have been identified. Time constraints, inadequate training, perceived lack of importance, lack of clear referral sources, and legal, economic and administrative complexities create barriers to physicians 
recording occupational histories. These factors have been identified by a number of studies [14-17].

Cognisant of the yet unsolved problem of the lack of occupational exposure surveillance data, another project was formulated. It is the subject of this manuscript. This project has taken into account the previous attempts to engage primary healthcare clinicians, and the research that has helped identify the barriers. The study made the commitment to learn from the previous initiatives how important it is to build relationships, identify the key questions, and be responsive to the needs and context-specific issues of the different clinician groups. We also made the commitment to make any survey short and easy to use which has been identified as a need for exposure assessment surveys $[18,19]$.

This project built on a pilot study that has been previously reported [20]. The long-term objective of the study was to determine a way to integrate the collection of occupational information as part of the routine clinical practice in health centres. In the shortterm, instead of focusing on the need for surveillance data, the emphasis was placed on the potential health benefits of screening patients for workplace risks and hazards. If clinicians were knowledgeable about their patients' work-related risk factors, this might have a positive impact on their patients' health. It could help identify existing work-related health problems and would facilitate conversation about prevention. These questions would 'complete the picture' that clinicians have of their patients. ('Completing the Picture' became the name of the study).

\section{Methodology}

\subsection{Research question}

The research questions that framed the research project were:

- Whether it was possible and feasible to collect information about occupational hazards within primary care;

- The logistical issues for collecting the information, including the facilitators and barriers;

- The possibility of adding these questions and answers to the patients' EMRs; and

- The major logistical issues to setting up widespread collection of 'real-time' exposures from workers across Ontario's primary care group practices, as well as eventually across Canada.

\subsection{The study, funder, and ethics}

This project was less about the actual responses to the questions on present-day occupational exposures, and more about the feasibility of clinicians asking questions on this topic during routine primary care visits, their comfort in asking these questions, and whether they would do anything with the answers. The study was funded by the Canadian Cancer Society Research Institute and the Canadian Institutes of Health Research. Ethical approval was granted for the project from the Waterloo-based Community Research Ethics Office.

\subsection{The research team}

The project was a collaboration between four research institutions and two occupational health and safety organizations. The research institutions were: the Occupational Cancer Research Centre at Cancer Care Ontario, the Centre for Research Expertise in Occupational Disease at the University of Toronto and St. Michael's Hospital, the Centre for Research Expertise in Musculoskeletal Disorders at the University of Waterloo, and the School of Rural and Northern Health at Laurentian University. The occupational health and safety organizations were the Occupational Health Clinics for Ontario Workers (OHCOW) and the Public Services Health and Safety Association.

The multidisciplinary research team included a qualitative researcher with experience in the practice and research of knowledge transfer and exchange, an occupational physician who has led previous studies on occupational exposure and making the link between exposure and cancer in clinical settings, a kinesiologist with expertise in workplace-based quantitative research into the prevention of musculoskeletal disorders, two epidemiologists with occupational exposure expertise, and an occupational hygienist. The project was managed by a knowledge broker with a background in health promotion and qualitative research.

\subsection{Organization Implementation Conceptual Model}

The Organization Implementation Model (see Fig. 1) was integral to the framing of this project. It helped: 
1. Identify the lack of knowledge about workplace exposures and risk factors and hence informed the survey questions;

2. Formulate the core components of the knowledge transfer and exchange intervention;

3. Adapt the interventions to the health centres' contexts and needs;

4. Direct the interactive nature of the engagement with the health centres;

5. Determine the questions in the semi-structured interview guide;

6. Frame the barriers and facilitators to implementing the survey;

7. Structure how the qualitative data would be analyzed; and

8. Organize the evaluation.

The conceptual model was developed specifically for workplace-based, knowledge-translation occupational health and safety initiatives [21-24]. The constructs included in the model are described in Table 1.

\subsection{Context of health centres}

In total, six health centres with patient-centred models of primary care practice were chosen. The first health centre trial became the pilot that we have reported on [20], and five others made up the trial that is the focus on this study. We defined a health centre as a member of the Association of Ontario Health Centres (now called the Alliance for Healthier Communities), which represents community-governed primary healthcare organizations, such as community health centres and nurse practitioner-led clinics.

These types of health centres have multidisciplinary teams to provide holistic care to patients, from primary care clinics to health promotion initiatives. Physicians are salaried instead of paid on a fee-forservice basis, and typical appointments with patients are 30 minutes long; longer than a typical medical appointment within a fee-for-service clinic. These health centres have a mandate to address the social determinants of health, of which work and working

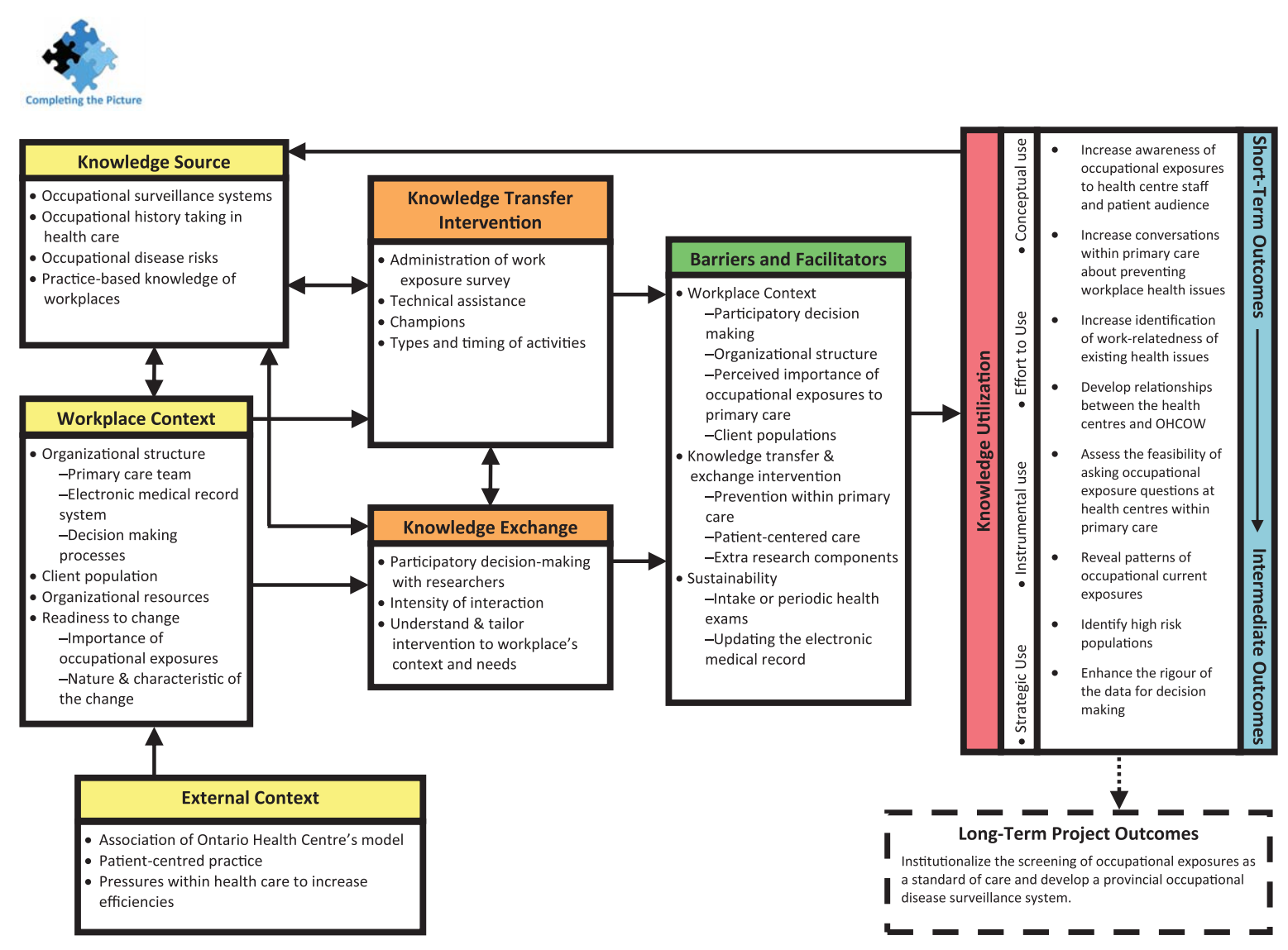

Fig. 1. Organization Implementation Model. 
Table 1

Organizational Implementation Conceptual Model constructs applied to the feasibility of clinicians asking patients about their exposure to occupational hazards project

\begin{tabular}{|c|c|}
\hline Conceptual Model Construct & Description of the Construct \\
\hline Knowledge Source & $\begin{array}{l}\text { Evidence-based knowledge about occupational surveillance systems, occupational history } \\
\text { taking in primary care, and occupational disease risks. This is matched with the unique } \\
\text { practice-based knowledge of the workplaces. }\end{array}$ \\
\hline Workplace Context* & $\begin{array}{l}\text { (1) organizational structure included (a) primary care team (b) electronic medical record } \\
\text { system, and (c) decision making processes; (2) patient population; (3) organizational } \\
\text { resources; (4) readiness to change included (e) importance of occupational exposures within } \\
\text { primary care, and (f) nature \& characteristic of the change. }\end{array}$ \\
\hline External context* & $\begin{array}{l}\text { External, relatively unchangeable factors that have an impact on the workplace's context in } \\
\text { relation to priorities and capacities within primary care. These include the healthcare and } \\
\text { organizational model of the health centres who were recruited for the study, patient-centred } \\
\text { care, and pressures within health care to increase efficiencies. }\end{array}$ \\
\hline $\begin{array}{l}\text { Knowledge Transfer Intervention; } \\
\text { Knowledge Exchange* }\end{array}$ & $\begin{array}{l}\text { The knowledge transfer and exchange intervention is explained fully in Section } 2.7 .3 \text { and Table } \\
\text { 3. The research team approached their interactions with the workplaces in a participatory, } \\
\text { pro-active manner and endeavoured to understand and tailor the intervention to each } \\
\text { workplace's context and needs. The workplaces provided local knowledge, and explored the } \\
\text { feasibility of embedding occupational exposure screening questions in their work. }\end{array}$ \\
\hline Knowledge Utilization & $\begin{array}{l}\text { Knowledge utilization was categorized as conceptual use, effort to use, instrumental use, or } \\
\text { strategic use. Conceptual use and effort to use are short-term outcomes, while instrumental } \\
\text { use and strategic use are intermediate outcomes. }\end{array}$ \\
\hline Short-term to Intermediate Outcomes & $\begin{array}{l}\text { Short-term outcomes include increasing awareness of occupational exposures to health centre } \\
\text { staff and patient audience, increasing conversations within primary care about preventing } \\
\text { workplace health issues, increasing identification of work-relatedness of existing health } \\
\text { issues, and developing relationships between the health centres and OHCOW, and assessing } \\
\text { the feasibility of asking occupational exposure questions at health centres within primary } \\
\text { care. Intermediate outcomes include revealing patterns of current occupational exposure, } \\
\text { identifying high risk populations, and enhancing the rigour of the data for decision making. }\end{array}$ \\
\hline Long-term Project Outcome & $\begin{array}{l}\text { Institutionalize the collection of occupational exposure information as a standard of care and } \\
\text { develop a provincial occupational disease surveillance system. }\end{array}$ \\
\hline
\end{tabular}

*These constructs are the focus of this article.

conditions are included. The health centres provide care to marginalized priority populations throughout the province, including new immigrants and refugees, people without health insurance, and are often located in low income or under-resourced communities.

The researchers chose these models of primary care because their patients are usually more marginalized, and would include vulnerable workers. Further, the researchers anticipated that focus on the social determinants of health, the longer appointment times, and the resources introduced by the multidisciplinary teams might mediate some of the challenges previously described by other studies on collecting occupational exposure information within primary care.

\subsection{Recruitment of health centres}

Recruitment of the six health centres (one for the pilot and five for this follow-up trial) was done with the help of two occupational health and safety organizations and through the research team members' connections. It usually involved emails, phone calls, and a number of face-to-face meetings at the health centres with administrative and clinical staff. Efforts were made to recruit health centres from geographically different areas of the province to reflect the diversity of work in urban and rural centres and in different regions in the province. See Table 2 for a summary of the characteristics of the five health centres that are the focus of this manuscript.

A number of concerns were expressed by clinicians during the recruitment process. Some of these concerns were the same ones brought up in the project's pilot study [20]. For example, clinicians were concerned about how much time the survey would take, their ability to address concerns about workplace hazards, and whether they may be deemed liable since they had learned about issues in a patient's workplace and had not acted to intervene. One health centre mentioned concern about whether they might compromise a patient's ability to get personal health insurance if work exposure was entered into their EMR. We addressed these concerns during the recruitment process. Only some of these concerns remained as themes in the final interviews with staff. 
Table 2

Summary of characteristics of and engagement with health centres

\begin{tabular}{lccccc}
\hline & Health Centre 1 & Health Centre 2 & Health Centre 3 & Health Centre 4 & Health Centre 5 \\
\hline Region in Ontario & Hamilton, Halton \& Brant & Hamilton, Halton \& Brant & Southwest & Southwest & Northeast \\
Urban/Rural & Urban & Urban & Rural & Urban/rural & Rural \\
Number of Surveys & 51 & 36 & 97 & 98 & 84 \\
Length of Engagement* & 7 months & 11 months & 7 months & 24 months & 12 months \\
\hline
\end{tabular}

*From first face-to-face recruitment meeting with health centre administrators and clinicians to interviews at the end of the survey trial.

\subsection{The work exposure survey intervention}

\subsubsection{Pilot study of work exposure survey intervention}

This knowledge transfer and exchange, knowledge broker-led intervention built upon the lessons learned in the pilot study of this project [20]. As a result of findings from the pilot, a number of changes were made to the intervention prior to the survey being subsequently trialed in these five health centres. Due to these changes, data from the pilot trial has not been included in this article.

The changes included the following:

- More time was spent to ensure that the clinical staff at the health centres were engaged with the study and a project champion amongst the clinical staff was identified;

- The work exposure survey was reduced from three pages to one page and only eight questions;

- More time was spent discussing what process would be used to distribute the surveys and collect and input the data into the EMRs;

- The health centres were offered the expertise and potential referral to the OHCOW; and

- Fact sheets were produced on different exposures, their potential health impact, and prevention strategies in the workplace.

\subsubsection{Work exposure survey}

Building on the findings from the pilot, a much shorter work exposure survey was developed by the research team (see Fig. 2). This basic screening tool was designed for healthcare clinicians to explore information about their patients' jobs and workplace exposures.

The eight questions asked about job title, job tasks, and then five specific exposures (noise, chemicals, heavy lifting, repetitive movements/awkward body positions, and asbestos), and a general question inviting the patient to discuss any other concerns about their work. The focus of the project was on the feasibility of asking questions, and not on the actual responses. Hence the survey was brief and was meant to facilitate conversation between clinicians and patients about the prevention of work-related conditions and improve the accuracy of diagnosis of work-related health issues.

\subsubsection{The work exposure survey implementation trial}

The knowledge transfer and exchange intervention had some core components common across all five health centres. A knowledge broker who represented the research team managed the project. She (RK) had ongoing engagement with the health centres throughout the trial including: in-person recruitment meetings; in-person meetings and communications to develop the health-centre-specific process map; the training and survey launch meeting; check-in meetings, emails, or phone calls; and conducting some of the final evaluation interviews.

The knowledge broker also wrote a report for each of the centres on the results of the implementation, and designed an infographic to be posted in the waiting room. The reports included the summary of survey data collected by their health centre, a comparison of their survey data with the average from all the health centres, and preliminary findings from exit interviews at all of the health centres. Each of these components of the intervention were considered positive opportunities to intensively engage with the health centre administrative staff and clinicians about the study.

Knowledge transfer and exchange interventions need to have a strong core with certain components that are common to all the targeted interventions in order to ensure integrity, fidelity, and hence comparability. However, details of the intervention can, and should, be adapted to fit to the needs of local settings [25, 26]. Adapting to the local context demanded many differences across the five interventions. Table 3 describes both the core implementation components and these differences (called "the adaptable periphery of an implementation" in the knowledge transfer and exchange literature [27]). For example, the length of engagement between the 


\section{Client Survey on Current Work Exposure}

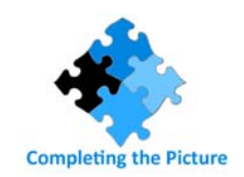

Date:

$$
(\mathrm{MM} / \mathrm{DD} / \mathrm{YY})
$$

By completing this survey you are indicating that you have read and understood the Client Survey Information and Consent Letter, have retained a copy of that letter and are agreeing to voluntarily participate in this research.

1. Are you currently working, or have you worked in Canada in the last 6 months? If yes, continue to Sections A \& B

$\square$ Yes $\square$ No

\section{Section A. Please refer to your current job or most recent main paid job.}

2. Job title/occupation:

3. Type of industry (ie. hospital, auto parts plant):

4. Do you work with chemicals?

$$
\square \text { Yes } \square \text { No }
$$

5. Is your workplace noisy?

6. Do you do heavy lifting?

7. Do you do repetitive movements or work in an awkward position?

8. Do you have any other concerns about things at work?

\section{Section B. Please refer to both current and past jobs.}

9. How frequently do you have contact with asbestos?

$$
\square \text { Never } \square \text { Rarely } \square \text { Occasionally } \square \text { Most days } \square \text { All days } \square \text { Don't know }
$$

\section{Section C. To be completed by the provider.}

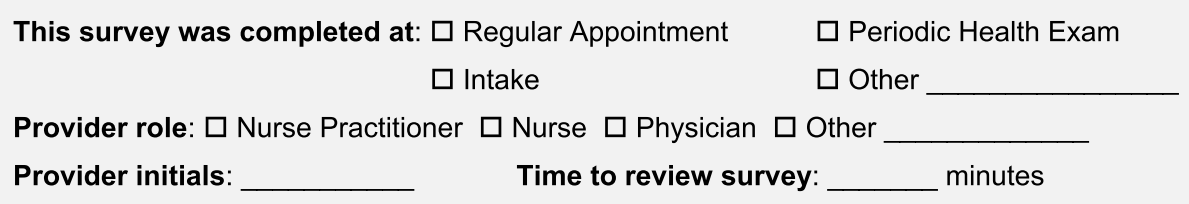

Fig. 2. Patient/Client Survey on Current Work Exposure.

research team and the five health centres varied considerably. Engagements ranged from seven to 24 months from the first face-to-face meeting with the clinical team and administrators, focusing on recruiting the health centre to the project, to the completion of the interviews with health centre staff after the trial.

While the sustainability of adding these occupational exposure questions to routine clinical practice was the long-term goal of the study, the researchers 
Table 3

Core components of the intervention and adaptations required by individual health centres

Core components
Clinical team members were invited to an initial presentation about
the project before the health centres agreed to participate. A
representative from OHCOW also presented at this meeting to
talk about their role and support services, including patient
referrals.
The health centres received a CAN $\$ 9,000$ stipend for the study, and
an additional CAN $\$ 1,000$ went to cover $\$ 10$ gift cards that were
tokens of thanks for up to 100 patients who agreed to complete
the survey.
The knowledge broker worked with members of the administrative
and/or clinical team to determine a process to fit the work
exposure survey into routine clinical practice. A formal process
map was drafted and shared with the health centre.

The work exposure survey included core components:

- Two job-related questions and six exposure questions

- Section (C), completed by clinicians when reviewing the survey, identified the type of health care appointment the survey was being reviewed at

An administrative champion was identified to help organize logistics and implement the intervention. This was often the clinical services director. A clinical champion was identified as well, and their role was to maintain interest and momentum in the intervention.

An orientation meeting with the entire clinical team was held at each health centre. This included a review of the survey process and a second presentation from OHCOW. The presentation included basic information on using the survey in clinical practice.

Four fact sheets were developed by the research team and provided to the health centres. They were given out to patients for their personal use or to use to facilitate discussion at patients' workplaces.

The knowledge broker was in contact with the health centres over email, phone, and/or in person throughout the duration of the intervention.

The patients were recruited by administrative staff. Patients were 16 years or older and had worked in Canada in the last six months. Patients reviewed an information letter about the study and a consent form. The consent was an implied consent. Completing the survey was taken as a sign of consent.

Clinicians (physicians, nurse practitioners, and occasionally nurses) reviewed the survey answers with patients. Patients received a $\$ 10$ gift card as a token of thanks.

Health centres trialed the survey for a minimum of three months, or until 100 surveys were completed.

After the trial was completed, researchers invited administrative and clinical team members to be interviewed as part of the final evaluation of the effectiveness of the intervention.

The results of the intervention were presented to each health centre. This included the health centre's work exposure data in comparison to aggregated health centre data, and preliminary results from interviews with all the health centres.
Adaptable periphery

Where the gift cards were purchased from was determined by the health centre. This ranged from grocery stores to coffee chops.

The health centres determined who was invited to this meeting. The knowledge broker encouraged involvement of the clinical team to increase buy-in. Participation varied from all the clinical staff, to representation from clinical staff, to only administrative staff. The process maps included variations of the following components:

- Who recruited the patients

- Which appointments were targeted for recruitment

- How the survey was completed

The work exposure survey included some adaptable components:

- Health centres were able to add additional occupational exposure questions that reflected their patient population. Two health centres added additional questions about dust, dust/fumes, and vibration.

- Over time Section (C), for clinicians to complete, included more information, such as the role of the clinician who reviewed the survey, and time it took to review the survey. Specific appointment types and clinician roles were modified to reflect the health centre's language and process map.

The clinical champions had varying levels of enthusiasm and personal buy-in to the project.

OHCOW offered additional training sessions for all health centres. An additional presentation was requested by and occurred at one health centre.

How the fact sheets were used and made available to patients was determined by the health centre.

Frequency and duration of contact was determined as needed, from bi-weekly to monthly.

Health centres that were unable to reach 100 filled-in surveys within three months could choose to extend their trial. 
Interviewer to complete this section

Date:

Location:

Survey

1. What was your experience of administering the survey?

Probe: What were the challenges? What went well?

2. What other information related to a client's work would be useful to know?

Probe: Do you think more detailed information would be useful? Duration or intensity of exposure? How useful was this information?

\section{Sustainability}

3. What would it take to make this part of the routine clinical care?

Probe: Do you have any suggestions on what would improve the survey?

4. How would the survey fit into routine care?

Probe: Who would administer it? How/Where would the information be stored within the EMR?

What type of appointments would these questions be asked at?

5. How important do you think it is to ask clients about what they are exposed to at work?

\section{Conclusion}

6. Is there anything else that we didn't talk about that you think we should know? Is there anything you would like to add?

Fig. 3. Example Interview Guide: Providers Administering Survey.

recognized that there were also components of the intervention that were necessary because it was a research project. These components would not normally be present in routine clinical practice. The additions included a paper and pen survey without the option of an electronic survey, consenting patients to participate in research, adding a scanned copy of the survey to the EMR, recruiting patients who fit the inclusion criteria, providing patients with a $\$ 10$ gift card as a token of thanks for their participation, and administering the gift cards.

\subsection{Evaluation of the interventions}

The following evaluation of the intervention is based upon the cross-case analysis of the qualitative interviews that were conducted at the five health centres at the termination of the survey trials to determine the barriers and facilitators facing clinicians asking patients about their exposure to occupational hazards.
Both administrators and clinicians were invited for one-on-one interviews. This included clinical team leads, health centre leadership, reception staff and other administrative team support staff, physicians, nurse practitioners, nurses, and other support staff. The interviews were semi-structured and guided by key questions and probes (see Fig. 3 for an example interview guide). They were conducted by two members of the research team (RK, DMK).

The analysis was also informed by five final feedback presentations to the health centres on the results of the survey trial. These presentations were delivered in-person at all-staff meetings and were an opportunity for the health centre staff to do a final reflection of the intervention.

\subsubsection{Interview data collection}

The interviews were mostly conducted in-person at the health centres, although three highly involved staff who were unable to be interviewed in-person 
were interviewed over the phone. Informed written consent was obtained from all participants prior to the interview and confidentiality was ensured.

Between eight and 15 interviews were conducted at each health centre. Variation in the number of interviews was a result of variation in the size of the health centre staff. There were 54 interviews in total for 53 staff persons. Of the interviewees, 36 were clinicians and 17 were administrators, which includes both administrative leadership and reception or support staff. If an interviewee held both clinician and administrative duties, they were included as a clinician. The interviews spanned from 4 minutes to 66 minutes long and totalled 977 minutes of audio recording. The interviews varied in length depending upon how engaged the person had been with the work exposure survey trial.

Interviews were digitally recorded and transcribed verbatim. The quality of the transcript was checked against the original recording by a research team member. All data was stored in password-protected and highly secure Cancer Care Ontario servers. Access to data was restricted to appropriate members of the research team.

\subsection{Survey data analysis}

The survey data analysis was based on 366 surveys collected at the five clinics. Survey data was entered into Microsoft Access. Descriptive analyses for each health centre's survey data were performed using SAS 9.4 software (SAS Institute Inc., 2013, Cary, NC, USA). Frequencies and crosstabs were generated for variables based on self-reports of occupation, industry, and a number of exposures.

\subsection{Interview data analysis}

The interview data analysis was based on the 54 interviews. The data were analyzed using deductive and inductive codes, and compared and contrasted using a matrix-based cross-case analysis with the health centre as the unit of analysis. The directions for a framework driven cross-case analysis, as outlined by Miles et al. (2014), was followed [28]. The analysis was based on a pre-determined conceptual model which informs the deductive analysis approach. These constructs were used as a priori thematic codes. When new themes or constructs emerged from the data they were also incorporated into the ongoing iterations of the conceptual model. This is a method that has been used by this research team for other projects and examples of matrices can be found therein [23, 29].

The primary analysis was conducted by one member of the research team for consistency (RK). The analysis went through the following eight steps:

1. Listening to the recorded interviews.

2. Creating an initial matrix for each of the five health centres. The columns were the job categories of the interviewees (ie. lead administrator, nurse, etc). The rows were the themes identified in the foundational conceptual model. As other themes emerged from the data, they were added as additional rows.

3. Uploading the transcripts to NVivo10 to conduct thematic coding and get a first-level deductive analysis.

4. Merging the five health centres' matrices into one matrix where the columns were the health centres and the rows were the themes. Enhancing the matrix to reflect the deductive reasoning.

5. Engaging in ongoing intensive discussions with the research team.

6. Synthesizing the deductive analysis, the matrix, and the intensive discussions with the research team.

7. Updating the matrix and determining the dominant emerging themes.

8. Recreating the conceptual model to reflect the new learnings for this project (Fig. 1; described previously).

At the final presentations to the health centres, when the researchers reported back to them on the results of the survey trial, the same questions were asked as had been asked during the final interviews. It was an opportunity for the clinicians to reflect once again on the feasibility of asking work exposure questions during their normal clinical practice. These discussions provided particular insight around the question of sustainability. With more distance from the survey trial, clinicians were able to comment on if, or how, they have integrated the survey questions into their routine practice. The researchers took notes on the discussions. These notes and reflections have been included as part of the analysis of the interviews.

\section{Survey results}

Table 4 includes a summary of the industry sectors of the 366 patients who completed the work 
exposure survey across the five health centres. Health and social services was the most common industry sector.

Table 5 summarizes the different exposures patients reported at their current jobs. These questions were asked as present-day ever/never (yes/no) questions. Repetitive movements/awkward body position was the most commonly reported exposure, with two thirds of patients reporting exposure. About half of patients reported noise exposure and doing heavy lifting on the job. Chemical exposure was the least common, but still quite high, at $44 \%$ of patients reporting the exposure.

Table 6 summarizes self-reported asbestos exposure from all jobs. The asbestos-exposure question was for both present and historical exposure, with more specificity on the exposure level with the answers given on a likert scale (all days/most days, occasionally, rarely, never). More than half of patients reported never being exposed to asbestos.

Table 4

Patients came from different industry sectors

\begin{tabular}{lcc}
\hline INDUSTRY SECTOR RANKING & Frequency & Percentage \\
\hline Health and social services & 60 & 16.4 \\
Manufacturing & 39 & 10.7 \\
Accommodation and food services & 34 & 9.3 \\
Construction & 33 & 9.0 \\
Business services & 29 & 7.9 \\
Retail trade & 28 & 7.7 \\
Educational services & 26 & 7.1 \\
Public administration & 20 & 5.5 \\
Agriculture & 19 & 5.2 \\
Transportation & 19 & 5.2 \\
Other & 59 & 16.1 \\
\hline
\end{tabular}

Table 5

Patients reported different exposures at their current job

\begin{tabular}{lcc}
\hline Exposure & Frequency & Percentage \\
\hline Chemicals & 160 & 43.7 \\
Noise & 199 & 54.4 \\
Heavy lifting & 187 & 51.1 \\
Repetitive movements/ & 243 & 66.4 \\
awkward body position & & \\
\hline
\end{tabular}

Table 6

Patients reporting asbestos exposure from all their jobs

\begin{tabular}{lcc}
\hline Asbestos Exposure & Frequency & Percentage \\
\hline All days/most days & 5 & 1.4 \\
Occasionally & 21 & 5.7 \\
Rarely & 43 & 11.7 \\
Never & 214 & 58.5 \\
Don't know/missing & 83 & 22.7 \\
\hline
\end{tabular}

\section{Interview results}

The Interview Results section is being presented in three topic areas, following the constructs of the Organizational Implementation Conceptual Model: (4.1) the workplace context; (4.2) the knowledge transfer and exchange intervention; and (4.3) sustainability.

1. Under the Workplace Context, we discuss participatory decision-making, organizational structure, perceived importance of occupational exposures to primary care, and the patient population.

2. Under the Knowledge Transfer and Exchange Intervention we discuss prevention within primary care, patient-centred care, and the extra research components of the intervention.

3. Under Sustainability, we discuss whether intake or periodic health exams are the right time to ask occupational exposure questions, and whether updating the electronic medical records would be helpful.

\subsection{Workplace context}

\subsubsection{Participatory decision making}

Researchers required the approval of the health centres' administrative teams in order to gain access to the clinical team. However, the clinical team and individual clinicians needed to buy-in to the project in order for the work exposure survey to be implemented. Clinicians are autonomous practitioners who consider their primary responsibility to their patients. Most of the health centers required consensus from their clinicians before agreeing to participate in the project. They involved them in discussions on how the work exposure survey would be administered. They were co-creators of the process maps. An administrator commented, The staff that were going to do it were involved in the process design. That's all I really cared about. If they weren't involved it would be very awkward for somebody in my role who has administrative responsibility to expect them to do something extra that they weren't necessarily part of. It's just very difficult to get buy-in. But people were included and if they said it was ok with them, go for it.

\subsubsection{Organizational structure}

The health centres that were recruited for this project had a team-based approach to health care 
and had access to additional resources that are not necessarily available in fee-for-service health care. This includes longer appointment times with patients, access to multidisciplinary clinicians for on-site referral support, and health promotion teams. This team-based approach supported the creation of the process maps that integrated the work exposure survey into the clinical practice (see Fig. 4). A nurse practitioner commented, We have fabulous resources here. We have counseling. We have women's groups. We have men's groups. Right now they' re implementing some anxiety and depression groups that teach patients about their disease processes. So if I can use it as a means of hooking them into these great resources, absolutely I would do that.

Many of the interviewees spoke about their 30 minute appointments with patients as a necessity to address the complexity of their patients' health issues. They commented that even with 30 minutes, it was still difficult to get through all that they needed to cover with their patients.

To reduce the already-constrained appointment times, four of the five health centres decided that the reception staff would recruit the patients and the patients would complete the surveys in the waiting room. This reduced the length of time within the appointment dedicated to the survey. The clinicians reviewed the survey with the patients and answered their questions. A nurse commented, [The lead administrator] had the forms ready at the front. She had reception staff on board so the physician or the nurse practitioner didn't have to go scrambling in the morning because if that's the case then it probably wouldn't have got done either. They've got so much to do and all of that was organized very well for them so it was just a matter of reviewing the form and the information on it and using the rest of [the] allied health team as well to implement it.

\subsubsection{Perceived importance of occupational exposures to primary care}

The framing of the intervention implies that collecting occupational exposures should be regarded as an important part of taking a patient's health history, and that it is similar in nature to asking for family history or smoking status. The implementation literature has found that the level to which workplaces value a proposed change is a key component of whether an intervention is adopted or not. This concept is called "valence" [30]. It was important to explore how the clinicians perceived the importance of collecting occupational exposure information within primary care.

Among both clinicians and administrators, there was agreement that working conditions can have an important impact on patient health. Many clinicians mentioned that they already ask their patients what work they do. A nurse said, We' re all about this holistic approach. So if we' re talking holistic, you've got to look at the work life too. I think we focus a lot on family life, likely because a lot of our patients don't work. But there's that work life that plays a huge component as well. That work life also can play a component on your support systems and your finances and everything else.

The administrators at the health centres consistently saw the value in the work exposure survey as a way to encourage clinicians to have conversations with patients about their work. Generally they were more enthusiastic than clinicians on this topic. An administrator said, We should be asking those questions and how much their occupation can affect their health. People are at work often times more than they're at home and we ask a lot about home life but don't necessarily ask about occupational hazards and just kind of assume that the employer is taking on that role or responsibility, and just really not asking. Not always being aware of the risks. This was supported by an administrator who said, In the framing of things and social determinants of health, occupational health doesn't get as much attention. So I thought this was a neat way of elevating vigilance around occupational health matters that was also useful to our practitioners.

\subsubsection{Patient populations}

Some of the clinicians and administrators were surprised at how challenging it was to recruit working patients for the work exposure survey. The health centres have priority populations whom they serve, and this includes marginalized populations who are less likely to be working. Many of their patients who are of working age are on social assistance, have disabilities, and/or have mental illness and are unable to work. A physician commented, One of my biggest challenges in my patients was to find people who actually were employed. They just don't work. I would go days without anybody who was employed on my list. Now part of that is I tend to see a lot of chronicpain patients, disability patients, mental health. So those people, they' re not working.

Clinicians often commented on how complex their patients were. For clinicians, this means that even 


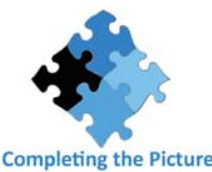

1. Client enters the health centre and shows their health card to the receptionist. The receptionist checks the client's EMR to determine eligibility for the survey. If the client is eligible for the survey and has not completed it, they receive a clipboard with the information letter/consent and survey.

2. Client reviews the consent form and survey in the waiting room. If they choose to, the client starts to complete the survey.

\begin{tabular}{|l|l|l|}
\hline YES & No & Appointment continues as usual \\
\hline
\end{tabular}

3. Client is invited to an exam room by a nurse. The nurse:

- Asks if the client has any questions about the consent form or survey.

- Answers questions about the consent form or survey.

- Updates the client EMR that they have completed/declined/do not qualify for the survey. This will be added in the lifestyle section of the EMR when the nurse is updating information on vitals.

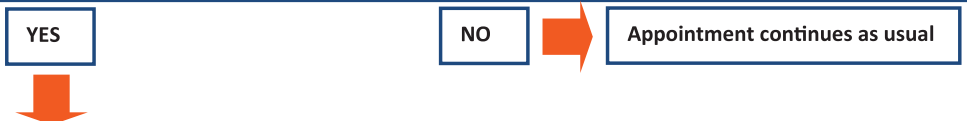

4. The nurse adds the client name and ID number to a sticky note.

5. Time permitted, the nurse practitioner or physician reviews the survey with the client. If there is not enough time, the provider flags the survey for review later.

6. At the end of the appointment, the client brings the paper survey to the receptionist and receives a gift card. The receptionist asks the client to initial for the gift card.

7. Receptionist scans the survey into the client's EMR.

- Surveys are entered into the EMR with a consistent file name for searchability.

- Paper surveys are stored together with the sticky note of the client name and ID removed.

8. Research team collects anonymized survey for analysis.

Fig. 4. Sample Process Map for Work Exposure Survey Trial at Health Centre.

though they have 30 minutes with their patients, the appointments were already constrained. Another physician reflected, My average patient has: diabetes, high blood pressure, abnormal lipids, a mental health diagnosis, a chronic pain diagnosis, narcotics, poverty, [and] family discord. Most of my patients come in with probably between five and 10 diagnoses a visit. So, although it sounds like half an hour is lovely - and that's the mistake that I made-that this will be a piece of cake, right? No. Not when you 
have 10 problems that you want to do well because you don't know when you're going to see the patient again.

At one health centre, there was some concern that their patients were disability-seeking and that they would use the work exposure survey as a way to get workers' compensation. A physician commented, There's concerns about sometimes triggering these questions. We do have a lot of people who are maybe looking to be off work. We have to be wary that people are malingerers. You know, they might say 'Yes, I have this!', and, 'Oh, I do have back pain' and then they' re going to want a note for off work. That kind of thing. So it's just going down a path that the appointment wasn't really intended for. However, the clinicians' concerns were not validated in their interactions with patients.

\subsection{Knowledge transfer and exchange intervention}

\subsubsection{Prevention within primary care}

The health centre model is better organized to support preventative health care, with an explicit focus on the social determinants of health and holistic care, than the fee-for-service health care system. Some clinicians found that the work exposure survey was an opportunity to speak with their patients about how to prevent future health issues that could result from their reported occupational exposures. A nurse practitioner said, For preventative health... I found the education piece was important. If we can prevent them from having or worsening carpel tunnel syndrome or things like that because we' re making them aware of things that exist, I feel like that's a very important part of our job.

However, some of the clinicians spoke about how challenging it was to do preventative health care. A physician identified lack of interest on the part of patients as an issue. I always, you know [in] regular exams, remind people about things like sun protection and hearing protection and that kind of thing. Using dust masks. That kind of stuff. I'll certainly bring those things up but the response is, 'Yeah, yeah. I know.' You know it's not, 'Oh tell me about that. I' $m$ very interested, I would like to learn more.' It's like, 'Yeah, yeah. Whatever.' That's the kind of response I would fairly frequently get.

Another physician highlighted all the competing demands on their time. We should know what our patients are doing. We should know what they are exposed to. But if I took all the things that my College says I' $m$ supposed to do, I can tell you that with 100\% of my patients [I would] never get everything done. Never. Because there isn't time. I can't do all the screening, and all the surveillance, all the history taking, and all the health teaching. There's just too much. You sort of hope to hit on important things over the years. But at any given time, do I have a $100 \%$ snapshot of that person? And then I'm also supposed to be doing things like end-of-life planning and safety things: 'Are you wearing seatbelts? Are you wearing your helmet? Drinking and driving? Do you have a will? Do you have a power of attorney? [... ]' There's hundreds of things on the list that I' $m$ supposed to be doing at any given time. You can't do it all. So, you pick and choose. So, occupational exposures don't hit the top ten usually.

A sub-theme of prevention within primary care is about preventing work-related health issues within primary care. A few clinicians spoke about how talking to patients about work is different from talking to patients about other lifestyle factors, such as diet or exercise. Patients have less control over changing a workplace compared to changing a personal lifestyle habit. As a physician said, So why am I not talking about occupational safety? Because a person's job is a lot harder to change. It's a lot more out of my hands and out of their hands than their smoking habits or their diet. And especially so in a lot of these manual labour jobs. They' re standing on a line, that's what they do. How do you change that? How do you go to your employer and say, 'My knees hurt, I want to sit down on the line.'? It's a lot easier to get a patient involved in quitting smoking than potentially losing his job. Because that's how they'll see it. 'If I complain I'll lose my job.' Makes sense. Occupational health is not just the patient and the physician. It's outside forces that are being imposed on the patient. Whereas the smoking, eating, the diet. That's the patient imposing that on himself.

Being employed and having an income is very important to patients, and for many, hazardous work exposures are tolerable until there is an acute health issue. The clinicians spoke about how patients were reluctant to do anything that might compromise their employment, and the clinicians were reluctant to suggest to their patients that they should do so.

Another physician highlighted that taking on workers' compensation is often a frustrating and not very productive process. In theory you might think okay you can identify these problems and do something about them. The reality for most of these patients is 
I've already spent many weeks and months and years trying to assist them through [workers compensation] and through various therapies, physiotherapy, massage therapy. Working with their employer, working with return to work advisors through [workers compensation], and ergonomists, to see if there are things we can kind of do to modify the type of work etcetera. Maybe I' $m$ a bit cynical about it but at the end of the day, many of these jobs are not things that the human body was designed to do for eight, or ten, or 12 hours a day and if you stick somebody on a factory assembly line asking to put widgets in a hole for ten hours a day or whatever, they have these injuries [...] And frankly, the workers' issues or symptoms or problems are relatively low concern because they'll find somebody else to do the job. You know it's a sad situation and I deal with it every day. So you know, getting back to your survey, I think there's value to it in some situations, but for many of these people, it's not the case that we haven't identified the problems, it's the case of they' re stuck in a job where they have limited work skills, limited training.

\subsubsection{Patient-centred care}

The interviews highlighted that clinicians speak with their patients about the relationship between work and health when their patients have a healthcare issue that the clinicians suspect may be related to work. This symptom-driven approach is aligned with patient-centred practice, which suggests that patients should be driving the conversations and priorities for health care in their lives. Clinicians focus on what their patient considers to be the most pressing issues.

Many clinicians said that when patients bring forward a health concern that the clinician suspects may be related to work, they will ask patients about work exposures. However, a few clinicians comments that although they consistently ask about occupational exposures for musculoskeletal issues, they are less likely to remember to ask about occupational exposures for a cough or hearing loss. A nurse practitioner added, I think it is important. I think we do sometimes miss that. For physical injuries, like a musculoskeletal injury, it's without a doubt that you're asking about work. I think I may miss it more if it's decreased hearing, but you know, people don't come in with that unless they're elderly. It's not very often. So if a young person did come in then it might be like, this is not generally normal so I may go in the direction, 'Well, what are you being exposed to?' But yeah, it absolutely is important. We certainly don't ask about work exposures every single time someone comes in.

A sub-theme of patient-centred care is the perceived usefulness of the survey in patient-centred care. There was variable engagement from patients with the survey information. Work exposure often was not their priority. As a physician said, Patients are here because they've got some specific issue they want to deal with and they're not really seeing this as a source of education around hearing protection or, you know... I always give them the spiel, but frankly, they're really not very interested in me telling them how to do their job.

As a nurse practitioner said, The patients that wanted to participate in learning and reviewing the handouts, I think they benefited from it. The ones that didn't, I think, you know, they might have been a little bit rushed or they wanted to focus on their personal health needs not pertaining to occupational hazards.

Although the researchers hoped that the survey would be a prompt to introduce new healthcare concerns, the interviews showed this was not the case. Most of the time, even when patients reported on the survey that they were experiencing occupational exposures, they said they did not have any new concerns about these exposures. A physician said, It didn't take a lot of time because, like I mentioned, I had no one identify, despite having exposures, any concerns. So no one was concerned about their exposures and no one really wanted to talk about it ... so we just went on with the other issues at hand.

Given this experience where few or no new health issues were introduced through asking the survey questions, a small number of clinicians directly commented that while asking the exposure questions may help a few patients over time, it wasn't helpful enough to become a routine part of care. As a physician said, I'm going to say $90 \%$ of the people didn't have an issue. So, you know, we are asking somebody to fill out a survey to identify that they don't have an issue. Then they come in and bring it to me to say, okay we don't have an issue. That's probably a waste of everyone's time in terms of expanding it.

This was supported by another physician who said, If people were being asked these questions at specific types of visits, I' $m$ sure on occasion something would arise. It might not be a problem but it could be a potential problem, or if they would have concerns, and it may be addressed early like in referring them off to whomever. So I guess that could be one benefit. But 
I think you would put in a lot of work for that [small] benefit. You'd have to do extra work for minimal acute benefit, let me put it that way.

\subsubsection{Extra research components}

Because the intervention was a research project, there were incentives (money for the health centres, gift cards for patients, and training on occupational exposures) provided to the health centres for their participation in the project. Incentives would not be present if the survey became part of routine primary care. This has implications because one of the goals of the study was to examine the feasibility of asking these questions as part of standard clinical practice. As an administrator noted, The money, I' $m$ not going to lie, it's a lot of money and it helped to get some of the things [we need]. We're getting some [training] and what not, so [we are getting] some educational use out of it. So that was definitely a bonus.

The gift cards to patients as a token of thanks for their participation was an incentive to patients to fill in the work exposure survey. There were mixed opinions about how important the gift cards were to the patients. Some providers thought the gift card was the main reason why patients were willing to complete the survey, while others though that patients were more interested in the survey itself. A nurse practitioner said, Many of them weren't interested [in having conversations about the survey] though. They just wanted their gift card.

As part of the study, OHCOW conducted training for the clinicians on using the work exposure survey and they offered their services as a referral to patients. Additionally, the research team prepared patient fact sheets on the exposures. These components were helpful to clinicians. An administrator mentioned that she thought the clinicians, were really happy to know that there are resources. That they don't need to be the expert. We were given those fact sheets right away and information about where you could send people if they had further questions. These resources were also appreciated by a nurse practitioner who said, We had the information to give patients if there's any concerns. We had a place that we could refer them to if there were concerns.

\subsection{Sustainability}

\subsubsection{Asking the survey at intake or periodic health exams}

Each of the health centres had control over how the survey questions were administered. Most of the health centres involved their reception staff in giving the surveys out to patients while they waited to be seen by the clinicians. Most of the clinicians and the administrators identified intake appointments (the initial appointment) and annual/periodic health exams (annual physicals) as the most promising opportunities to include work exposure questions into routine clinical practice. These types of appointments are ones where clinicians are able to drive the direction of the appointment, rather than primarily being responsive to the patients. Further, at these appointments, work status (whether or not patients are employed) is a routine question.

One nurse practitioner said, I think it would be fairly easy to integrate it into our practice. It's just another question that we ask at certain visits. I don't think it would be something that I would be asking every single visit. Probably during intakes and physicals. That's when I' m asking more questions than just the regular routine questions. Another commented, If you do it at an annual health review it's really good because you already ask about a bunch of different social determinants, so if you ask about their workplace exposures, it kind of flows in nicely.

However, it was noted that these annual/periodic health exams are no longer recommended. This presents a sustainability challenge. As a nurse said, With the [change] in guidelines [...] physicals aren't necessarily recommended anymore. [...] So I'm not really sure. I would have to really brainstorm where to input that for patients that have already done intakes.

\subsubsection{Updating the electronic medical record}

If occupational exposure questions were asked within intake appointments or annual/periodic health exams, it would be important to enter this information within the Cumulative Patient Profile (which has standard demographic questions) within the EMR. Including questions on work within regularly accessed and completed sections of EMRs would help remind clinicians to ask work exposure questions. Various EMR platforms include complex sets of questions for work, but many sections of the EMR are not routinely completed or referenced.

However, the health centres have different versions and platforms for their EMRs, and hence the data is not easily extractable. As a nurse said, When we do our intakes, in our EMR, there is a lifestyle section. It does ask about work. It just doesn' task about work in detail. But during an intake it could be incorporated. We ask questions about all kinds of stuff. So all it 
would take would be to add a section with a few more questions, more specific to what people do for work. A nurse practitioner added, We do have templates in our computer for annual health exams. [I don't know if work] could be part of the template so that when I go through I could fill [it] out. I should ask about that. If [work] was part of the template that would tweak our memories.

Additionally, not every clinician uses the EMR in the same way, so achieving consistent practice change within one health centre, or across the province, may be challenging. As physician said, I think social history is probably the [least] informed part of the [Cumulative Patient Profile]. It's not filled out as well as the other parts, right now. So that needs to be updated. [What should be updated is information] like marriage, single, divorced - that's all social-and work, occupational, retired, alcohol use, smoking. It's all part of the social fabric. And it's important. Lots of times it's lacking.

\subsubsection{Discussions at the final presentations on the results of the trial}

The researchers gave a presentation on the results of the survey trial to each of the health centres between three and nine months after the interviews. These feedback sessions were attended by clinicians and administrative staff and were interactive. The health centres also received the presentation slides, a report, and an infographic to post in the waiting room. Each researcher took notes at these presentations and together they reflected on key themes that emerged from the conversations. At these feedback meetings, clinicians and administrators commented that the results of the survey data were reflective of where they thought their patients worked and what they were exposed to at work. The questions seemed to have 'face validity'. For example, patients who worked in healthcare and in construction reported high exposure to ergonomic issues in comparison to other industries.

While sustainability was discussed during other meetings and in the interviews the researchers had had with health centre staff, no discussions occurred at these final feedback sessions about how the health centres had (or plan to) integrate the survey questions into their intake appointments or periodic health exams. The absence of this topic was noteworthy, given how much it was a focus of previous conversations, meetings, and interviews.

Some clinicians continued to indicate their reluctance to collect routine general work exposure information. They said their focus needed to be on their patients' health concerns, and within their patient populations this seldom related to work. At one health centre, clinicians spoke about their interest in a tool that could assess the work-relatedness of a particular health concern. For example, if a patient was presenting with asthma, clinicians were interested in a set of questions that would help determine if their patient's asthma was caused by or exacerbated by their work. This request had not emerged during the interviews.

At another health centre, clinicians spoke about how they perceived their patients' workers compensation claims were being denied at higher rates than previously. Clinicians said this had led to them being increasingly hesitant to file claims for patients, both in terms of raising their patients' expectations of being compensated and increasing their own workloads, without an expectation of positive return.

Interestingly, similar barriers have recently been identified by researchers at the Migrant Clinicians Network, based in Austin Texas [31]. They listed major barriers encountered by clinicians and community health workers at community health centres to providing occupational healthcare, including competing priorities, limited appointment time, and lack of training. They also mentions their frustration with accessing workers' compensation.

\section{Limitations}

Social desirability bias was a concern in this study, especially in regards to the interview question about the importance of asking patients about their work and workplace exposures. The researchers wondered if the interviewees felt obligated to respond more positively than they actually felt. The interviewees knew that the research was prioritizing occupational exposures, the health centres had received financial support to ask the questions, work is one of the (many) social determinants of health to which the health centres are publically committed to address, and there are detailed questions on work embedded in some of the electronic medical records that they could be asking. However, the reality is probably much more nuanced. Clinicians were often less certain about where questions for working conditions ranked in their prioritization of screening patients and addressing the social determinants of health. 
The researchers were unable to hear directly from patients about their experience of completing the survey and their interest in their clinician screening them for work exposures. This would have been a contribution to the research. However, the health centres found this request to be logistically difficult. The researchers relied on clinicians for their account of their interactions with patients. Given that the study was focused on organizational change at the health centre-level, this was an appropriate decision.

\section{Discussion}

The Ministry of Labour and the Occupational Health and Safety System in Ontario have identified the need for the systematic collection of occupational exposure and health data for ongoing surveillance and hence targeted prevention initiatives. This project responded to this high-level policy initiative. It investigated the feasibility of primary care clinicians collecting information on occupational exposures and entering it into patients' electronic medical records.

\subsection{The purpose of collecting information on workplace exposures}

A fundamental question that needs to be asked is: for what purpose do we want occupational exposure questions asked? To whose benefit is it to collect information on occupational exposures? Will this information: (1) help solve a present clinical problem; (2) help solve a clinical problem in the future, and hence could have prevention purposes; (3) create a surveillance system for the province to help direct its policy initiatives; or (4) help provide data for research?

No clear clinical purpose for asking basic work exposure screening questions emerged, and there was limited motivation to adopt the questions into routine clinical practice once the research trial ended. In their daily practice, clinicians are responsive to symptoms or complaints that are introduced by their patients. Although some clinicians spoke about the benefit of the survey in select appointments, and the ease of use of the survey, generally clinicians did not perceive the questions as applicable enough or useful enough to their practice - to their lived experience and daily clinical realities - to be prioritized, at least in routine, patient-initiated visits.

There are challenges to doing prevention in primary care, and the focus of providing patient-centered health care creates less opportunity for clinicians to introduce prevention-focused engagement into appointments. The answers to the work exposure questions were not helpful in addressing patients' presenting problems, and hence clinicians had difficulty prioritizing these questions. The clinicians stated that they did not find out about new health concerns through asking the survey questions. Further, generally even when a patient said they were currently exposed to noise, they said they were not concerned about their hearing, and the conversation moved on to the patients' present-day health concerns.

Moreover, the basic work exposure questions that were asked do not provide the quality of answers that are necessary for surveillance research, or to determine policy-prevention initiatives. Clinicians were very clear about time constraints within their appointments, and hence a longer surveillance survey that offers more research-relevant, or even policy-relevant answers, is not seen as a priority or feasible.

\subsection{Clinicians are asking work-related questions}

The study found that clinicians think that work and working conditions have an important impact on patient health. Further, clinicians are already asking about work status when they do an intake appointment or periodic health exam. They already ask work-related questions when patients have a health concern that clinicians suspect may be related to a work exposure. Hence clinicians commented that they already collect work-related information when they think it is appropriate and useful to providing care to their patients.

Patients' EMRs sometimes have a complex set of questions and fields related to work. The technology of the EMRs is not the issue. However, there is an issue in first, collecting work exposure data at health centres, and then sharing data across different health centres. Clinicians do not necessarily fill out EMR questions or fields consistently, and the EMR platforms across centres are not uniform. This presents issues for extracting the data for surveillance purposes.

\subsection{Taking the lead from patients}

Even when patients answered positively to the questions, they did not express concern to their clinicians about their exposures. If patients were 
experiencing a health issue as a result of exposures at work, clinicians already knew about the concern. Additionally, patients and clinicians were somewhat reluctant to explore prevention initiatives around workplace-based issues. Motivating patients to address harmful workplace exposures is a more challenging task than motivating patients to change other lifestyle risk factors, such as smoking or diet. Further, clinicians understood how important work was for these workers, and the positive impact of employment and income. Equally, clinicians were aware of the potential for patients being harassed or fired from work if they made a complaint. Both of these were perceived to be more important to the patients than potential future health risks from exposures.

In an atmosphere of patient-centred care, if patients do not introduce the topic of their work exposures, it is difficult to persuade clinicians to do something that is not immediately relevant to the clinical needs of their patients. Some clinicians also mentioned that in the past they had persuaded their patients to file for workers' compensation, but after a lengthy process, their claims were denied. This experience has contributed to both clinician and patient reluctance to pursue filing for workers compensation.

\section{Conclusion}

Multiple limitations and barriers were identified in this exploration of the feasibility of clinicians asking patients questions about occupational exposures as part of regular clinical practice. In the pilot study, clinicians mentioned a number of barriers that the research team tried to respond to with this trial. However, even when those barriers were identified and responded to, it did not make a difference in clinicians' perception of the relevance, usability or acceptance of the exposure questions into regular practice. Further, creating a tool for clinicians that would meet the data quality requirements for surveillance data would exacerbate many of the issues already identified by clinicians. In conclusion, considering all the barriers that have been identified, we may need to reflect on whether primary care clinical practice is the most appropriate place to collect more detailed work and exposure information.

\section{Acknowledgments}

This project received funding from the Canadian Cancer Society Research Institute (CCSRI) and the
Canadian Institutes for Health Research (CIHR), through a Prevention Research grant competition (awarded in 2014). The study received ethics approval through the Waterloo-based Community Research Ethics Office. The authors would like to extend their sincere thanks to the clinicians and administrators at the six health centres in Ontario who volunteered their time and knowledge to this study. We would like to thank Dr. Richard Wells, Dr. Nancy Lightfoot, Dr. Victoria Arrandale, and Ms. Irena Kudla for their expertise and help. We would also like to thank the Occupational Health Clinics for Ontario Workers and the Public Services Health and Safety Association for their collaboration and partnership on this study.

\section{Conflict of interest}

None to report.

\section{References}

[1] Del Bianco A, Demers PA. Trends in compensation for deaths from occupational cancer in Canada: A descriptive study. CMAJ Open. 2013;1(3):E91-E96. doi:10.9778/cmajo.20130015

[2] Workplace Safety and Insurance Board. [By the Numbers: 2016 WSIB Statistical Report; Schedule 2]. Toronto; WSIB; 2016. [cited 2017 Oct 16]. Available from: http:// www.wsibstatistics.ca/S2/Day\%20of\%20Mourning\%20_\% 20WSIB $\% 20$ By\%20The\%20Numbers_P.php

[3] Cancer Care Ontario, Occupational Cancer Research Centre. Burden of occupational cancer in Ontario: Major workplace carcinogens and prevention of exposure. Toronto: Queen's Printer for Ontario. 2017. Available from: http://www.occupationalcancer.ca/wp-content/uploads/ 2017/10/Burden-of-Occupational-Cancer-in-Ontario.pdf

[4] Tompa E, Kalcevich C, McLeon C, Lebeau M, Song C, McLeod K, Kim J, Demers PA. The economic burden of lung cancer and mesothelioma due to occupational and paraoccupational asbestos exposure. Occup Environ Med. 2017. doi: 10.1136/oemed-2016-104173

[5] Wells RP. Why have we not solved the MSD problem? Work. 2009;349(1):117-121. DOI: 10.3233/WOR-2009-0937.

[6] Kramer DM, Holness DL, Haynes E, McMillan K, Berriault C, Kalenge S, Lightfoot N. From awareness to action: Sudbury, mining and occupational disease in a time of change. Work. 2017;58(2):149-62. DOI: 10.3233/WOR-172610

[7] Coelho DA, Tavares CSD, Lourenço ML, Lima TM. Working conditions under multiple exposures: A crosssectional study of private sector administrative workers. Work. 2015;51(4):781-9.

[8] Farquhar A, Molino L. The Ontario Occupational Disease Strategy Framework. 2010. WSIB Ontario; [cited 2017 Oct 16]. Available from: http://www.ohcow.on.ca/edit/files/ news/16102015/FINAL\%202010\%20OD\%20Prevention\% 20Strategy.pdf 
[9] Ontario Ministry of Labour. Healthy and Safe Ontario Workplaces: A Strategy for Transforming Occupational Health and Safety. 2013 [cited 2017 Oct 16]. ISBN 978-14606-3281-9 (PRINT). Available from: https://www.labour. gov.on.ca/english/hs/prevention/strategy/index.php

[10] Wolfe V. Introduction to Ontario's Occupational Disease Action Plan: Aspirational and achievable. Occupational Disease Action Plan. Presentation. St. Michael's Hospital, Occupational Medical Rounds. September 27, 2017. St. Michael's Hospital, Toronto. [cited 2017 Oct 16]. Available from: http://www.ohcow.on.ca/ohcow-leadsontarios-occupational-disease-action-plan.html

[11] Kosny A, MacEachen E, Ferrier S, Chambers L. The Role of Health Care Providers in Long Term and Complicated Workers' Compensation Claims. J Occup Rehabil. 2011;21(4):582-90.

[12] Richards-Taylor A, Keay J, Thorley K. Do GPs record the occupation of their patients? Occup Med (Lond). 2013;63(2):138-140.

[13] Thorley K, Haigh R, Pearson A. Recording occupation in general practice - a second cycle audit. Occup Med (Lond). 2015;65(1):54-6.

[14] Thompson JN, Brodkin CA, Kyes K, Neighbor W, Evanoff B. Use of a questionnaire to improve occupational and environmental history taking in primary care physicians. J Occup Environ Med. 2000;42(12):1188-94.

[15] Harber P, Merz B. Time and knowledge barriers to recognizing occupational disease. J Occup Environ Med. 2001;43(3):285-8.

[16] McCurdy SA, Morrin LA, Memmott MM. Occupational history collection by third-year medical students during internal medicine and surgery inpatient clerkships. J Occup Environ Med. 1998;40(8):680-4.

[17] Holness DL, Tabassum S, Tarlo SM, Liss GM, Silverman F, Manno, M. Practice patterns of pulmonologists and family physicians for occupational asthma. CHEST Journal. 2007;132(5):1526-31.

[18] Bao S, Silverstein B. Categorizing job physical exposures using simple methods. Work. 2012;41(Supplement 1):39457. DOI: 10.3233/WOR-2012-0691-3945

[19] Ericsson P, Björklund M, Wahlström J. Exposure assessment in different occupational groups at a hospital using Quick Exposure Check (QEC) - A pilot study. Work. 2012;41(Supplement 1):5718-20. DOI: 10.3233/WOR2012-0929-5718

[20] Kushner R, Kramer DM, Holness DL. Asking clients at a community health centre about their occupational exposures: A knowledge transfer feasibility case study. SAGE Research Methods Cases. 2018. doi:10.4135/9781526428 677

[21] Kramer DM, Tenkate T, Kushner R, Strahlendorf P, Gardner A. and Holness DL. Sun Safety at Work Canada: A multiple case study protocol to develop sun safety programs and policies for outdoor workers. Implement Sci. 2015;(10)97. http://www.implementationscience.com/content/10/1/97 doi:10.1186/s13012-015-0277-2

[22] Kramer DM, Haynes E, Holness DL, Strahlendorf P, Kushner R, Tenkate T. Sun Safety at Work Canada: Baseline evaluation of outdoor workplaces recruited to participate in a sun safety knowledge transfer and exchange intervention. Safety Science. 2017;96:172-82. doi: 10.1016/ j.ssci.2017.03.011

[23] Haynes E, Kramer DM, Strahlendorf P, Holness DL, Kushner R, Tenkate T. A cross-Canada knowledge transfer and exchange workplace intervention targeting the adoption of sun safety programs and practices: Sun Safety at Work Canada. Safety Science. 2017;102:238-50. doi: 10.1016/j.ssci.2017.10.013

[24] Kramer DM, Cole DC, Leithwood K. Doing Knowledge Transfer: Engaging management and labour with research on employee health and safety. Bull Sci Technol Soc. 2004;24(4):316-30. http://journals.sagepub.com/ doi/abs/10.1177/0270467604267003

[25] Chambers DA, Glasgow RE, Stange KC. The dynamic sustainability framework: Addressing the paradox of sustainment amid ongoing change. Implementation Science. 2013;8:117. doi: 10.1186/1748-5908-8-117

[26] Moore JL, Carpenter J, Doyle AM, Doyle L, Hansen P, Hahn B, Hornby TG, Roth HR, Spoeri S, Tappan R, Van Der Laan K. Development, Implementation and Use of a Process to Promote Knowledge Translation in Rehabilitation. Arch Phys Med Rehabil. 2017;pii:S0003-9993(17)31085. PMID: 28928025. doi: 10.1016/j.apmr.2017.08.476

[27] Damschroder LJ, Aron DC, Keith RE, Kirsh SR, Alexander JA, Lowery JC. Fostering implementation of health services research findings into practice: A consolidated framework for advancing implementation science. Implement Sci. 2009;4:50. https://doi.org/10.1186/1748-5908-4-50

[28] Miles MB, Huberman AM, Saldana J. Qualitative Data Analysis: A Methods Sourcebook. 3rd ed. Thousand Oaks, California: SAGE; 2014. ISBN 978-1-4522-5787-7

[29] Kramer DM. Haynes E, McMillan K, Lightfoot N, Holness, DL. Iterative Method of Analysis of 90 Interviews from Two Communities: Understanding How Sudbury and Sarnia Reduced Occupational Exposures and Industrial Pollution. SAGE Research Methods Cases; 2018. doi: $10.4135 / 9781526443779$

[30] Weiner B. A theory of organizational readiness for change. Implement Sci. 2009;4:67. doi.org/10.1186/1748-5908-467

[31] Simmons JM, Liebman AK, Sokas RK. Occupational Health in Community Health Centres: Practitioner Challenges and Recommendations. New Solutions: A Journal of Environmental and Occupational Health Policy. 2018;0(0):1-21. https://doi.org/10.1177/ 1048291117749937 